\title{
5-Hydroxychromone, An Alternative Scaffold for Anti-HCV 1,3-Diketo Acid (DKA)
}

\author{
Chaewoon Lee, ${ }^{a}$ Kwang-su Park, ${ }^{a}$ Hye Ri Park, Jun Cheol Park, ${ }^{\dagger}$ Bokhui Lee, Dong-Eun Kim, and Youhoon Chong* \\ Department of Bioscience and Biotechnology, Bio/Molecular Informatics Center, Konkuk University, Seoul 143-701, Korea \\ *E-mail: chongy@konkuk.ac.kr \\ ${ }^{\dagger}$ National Institute of Animal Science, Rural Development Administration, Suwon 441-706, Korea \\ Received August 16, 2010, Accepted September 15, 2010
}

Key Words: Hepatitis C Virus (HCV), 5-Hydroxychromone, Enol-keto isostere, Antiviral effect

Chronic hepatitis $\mathrm{C}$ virus (HCV) infection, which affects more than 180 million patients worldwide, is one of the leading causes of cirrhosis and liver failure. Since the discovery of the HCV in $1989,{ }^{1}$, significant progress has been made towards the development of HCV therapy. Nevertheless, until now, combination of pegylated interferon $\alpha-2 \mathrm{a}$ and ribavirin is the only approved anti-HCV treatment, ${ }^{2}$ and the combination therapy suffers from low sustained antiviral response and significant side effect. ${ }^{3}$ Therefore, the combination drug doses and treatment duration are restricted and more effective and better-tolerated treatment options for HCV infection are urgently needed. ${ }^{4}$

1,3-Diketo acid (DKA, Fig. 1) is one of the potent anti-HCV agents. In particular, a DKA derivative with a 3,5-dichloro-2cyanophenylmethyloxy substituent at the meta position of the aromatic ring (1, Fig. 1) was reported to be a nanomolar (i.e., $45 \mathrm{nM}$ ) in vitro inhibitor of the HCV RNA-dependent RNA polymerase (RdRp). ${ }^{5}$ Previously, through molecular modeling ${ }^{6,7}$ and structure-activity relationship study, ${ }^{8}$ we have also identified that a 4-chlorobenzyloxy group substituted ortho to the aromatic ring provided the resulting aromatic DKA derivative with potent antiviral activity [2, Fig. $\left.1\left(\mathrm{EC}_{50}=0.54 \mu \mathrm{M}\right)\right]$. However, DKAs suffer from unfavorable physicochemical as well as pharmacokinetic properties such as chemical instability, irreversible covalent binding to protein and poor stability in plasma. ${ }^{9}$ As a result, in spite of potent inhibition of the target enzyme, most DKAs show suboptimal antiviral activity $\left(\mathrm{EC}_{50}=\right.$ $23 \pm 8 \mu \mathrm{M}$ for compound 1) in cell-based assay. ${ }^{10}$ Therefore, studies on the structural modification of DKAs have been focused on optimization of the antiviral activity in cell-based assay through extensive structure-activity relationship study. As the mode of action of DKAs is known to involve chelation of the metal ions present in the active site of the HCV RdRp, ${ }^{9}$ optimization of antiviral activity of DKAs has been actively performed through structural modification of the metal-binding 'keto-enol acid' unit of DKA. Isosteric replacement of the free carboxylic acid with triazole (S1360), ${ }^{11}$ tetrazole (5CITEP), ${ }^{12}$ pyridine $(\mathrm{L}-870,810),{ }^{12-15}$ and a neutral carbonyl group (Raltegravir) ${ }^{16}$ have been reported to give potent anti-HCV agents, and it was also found that even a 'monoketo acid' functionality (Elvitegravir, Fig. 1) can mimic the metal-binding function of the DKA. ${ }^{17}$

However, it has not been attempted to reverse the order of the metal-binding unit, 'keto-enol acid' or 'monoketo acid', to 'enol-keto' functionality. In this study, we reasoned that a 5-hydroxychromone would serve as an excellent scaffold for this purpose, and a novel 5-hydroxychromone derivative (3, Fig. 2) with 3-substituted benzyloxy and phenyl substituents at 6 and 7 position, respectively, would provide a structural mimic for anti-HCV DKA 2. The superimposed structure of $\mathbf{2}$ and $\mathbf{3}$ supports this idea by showing atom-by-atom match of the metalbinding units (shown in thick lines) as well as the aromatic ether linkage (Fig. 2).

Herein, we report synthesis of novel 6-arylmethyl-5-hydroxy7-phenyl-chromone derivatives (3) with various aromatic substituents $\left(\mathrm{R}=\mathrm{Cl}, \mathrm{Br}, \mathrm{CN}, \mathrm{NO}_{2}, \mathrm{CF}_{3}, \mathrm{Me}, \mathrm{OMe}\right)$ and evaluation of their anti-HCV activities.

Methylation followed by Suzuki coupling of the commercially available 5-bromovanillin (4, Scheme 1) provided 5,6dimethoxybiphenyl-3-carbaldehyde 6 in $83 \%$ combined yield. The benzaldehyde 6 was then oxidized by mCPBA to the co-

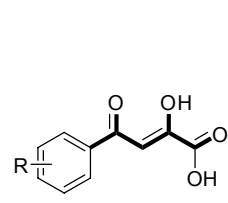

DKA

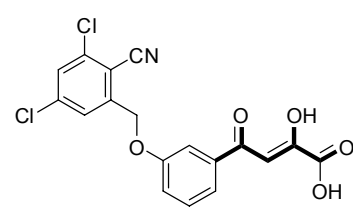

1

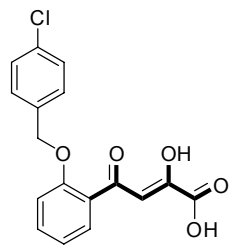

2

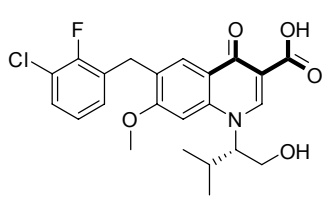

Elvitegravir

Figure 1. Structures of DKA, meta-(3,5-dichloro-2-cyanophenylmethyloxy) DKA (1), ortho-4-chlorobenzyloxy DKA (2), and elvitegravir. The metal-binding unit is represented as thick lines.

${ }^{\mathrm{a}}$ These authors contributed equally to this work. 

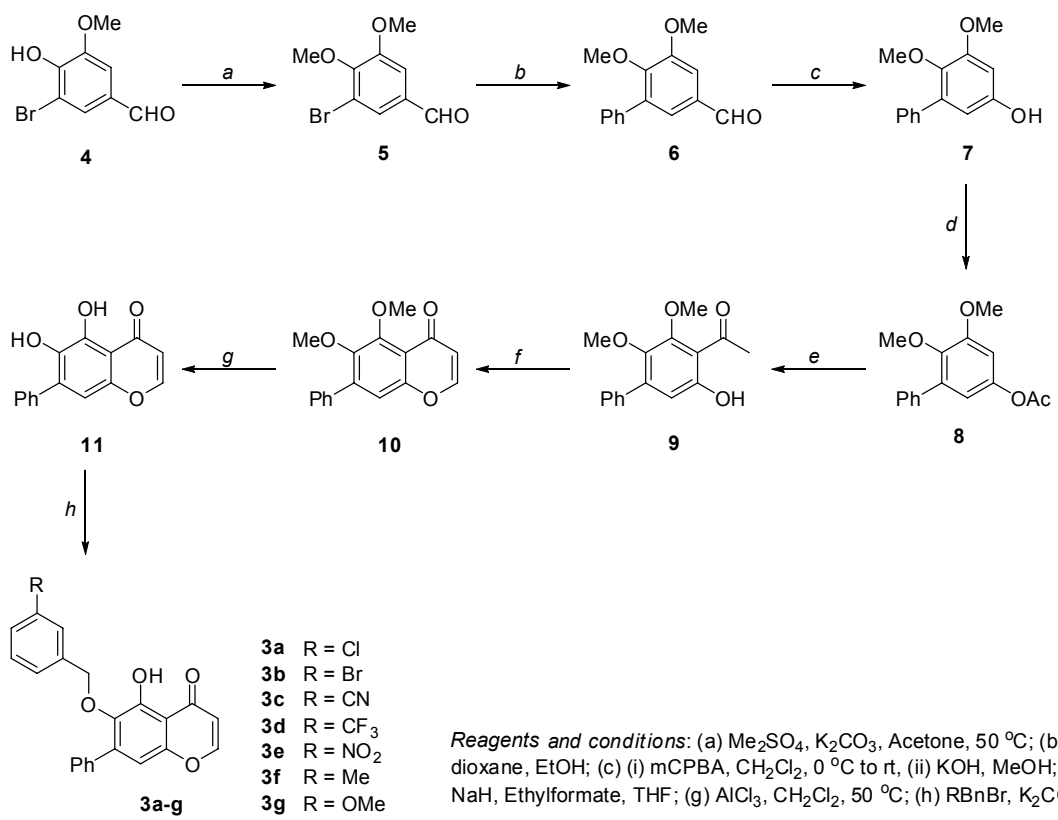

Reagents and conditions: (a) $\mathrm{Me}_{2} \mathrm{SO}_{4}, \mathrm{~K}_{2} \mathrm{CO}_{3}$, Acetone, $50{ }^{\circ} \mathrm{C}$; (b) $\mathrm{PhB}(\mathrm{OH})_{2}, \mathrm{Pd}\left(\mathrm{PPh}_{3}\right)_{4}, 2 \mathrm{M} \mathrm{Na}_{2} \mathrm{CO}_{3}, 1,4-$ dioxane, $\mathrm{EtOH}$; (c) (i) $\mathrm{mCPBA}, \mathrm{CH}_{2} \mathrm{Cl}_{2}, 0^{\circ} \mathrm{C}$ to rt, (ii) $\mathrm{KOH}, \mathrm{MeOH}$; (d) $\mathrm{AcCl}, \mathrm{Pyr}$; (e) $\mathrm{BF}_{3} \mathrm{Et}_{2} \mathrm{O}, \mathrm{AcOH}, 70{ }^{\circ} \mathrm{C}$; (f) $\mathrm{NaH}$, Ethylformate, THF; (g) $\mathrm{AlCl}_{3}, \mathrm{CH}_{2} \mathrm{Cl}_{2}, 50{ }^{\circ} \mathrm{C}$; (h) $\mathrm{RBnBr}, \mathrm{K}_{2} \mathrm{CO}_{3}$, acetone, DMF

Scheme 1. Synthesis of 6-arylmethyl-5-hydroxy-7-phenyl-chromone derivatives (3a-3g)

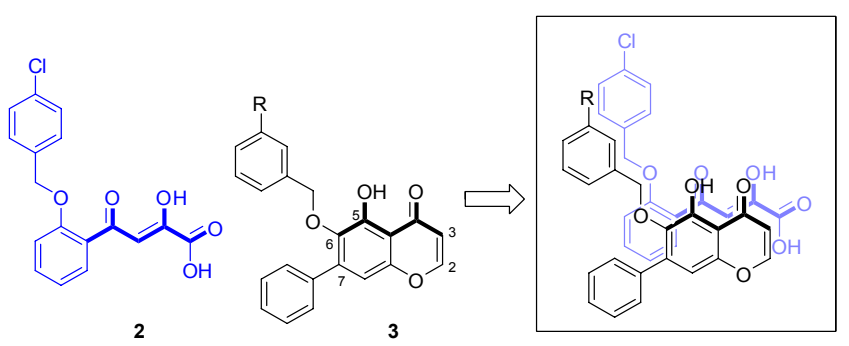

Figure 2. Superimposed structures of ortho-4-chlorobenzyloxy DKA (2) and 6-arylmethyloxy-5-hydroxy-7-phenyl-chromone (3) around the common metal-binding unit.

rresponding formate which, without purification, was hydrolyzed to the phenol 7 in $61 \%$ yield. The ortho-hydroxy acetophenone 9 was obtained by acetylation of 7 followed by Fries rearrangement ( $92 \%$ yield). Treatment of 9 with $\mathrm{NaH}$ and ethyl formate resulted in construction of the chromone scaffold to afford $\mathbf{1 0}$ in $90 \%$ yield. Lewis acid-promoted demethylation followed by alkylation with various 3 -substituted benzyl bromides provided the desired 6-arylmethyloxy-5-hydroxy-7phenyl-chromone derivatives (3a-3g) in $54-79 \%$ yield.

The synthesized hydroxychromone derivatives (3a-3g) were evaluated for their ability to inhibit HCV replication in Huh-5-2 cells, which was kindly provided by Prof. R. Bartenschlager, University of Heidelberg, Germany. ${ }^{18-20}$ The cytostatic effect of the test compounds was also evaluated in the same cell line. Antiviral effect and cytostatic effect are summarized as $\mathrm{EC}_{50}$ and $\mathrm{CC}_{50}$, respectively, in Table 1.

The 5-hydroxychromone derivatives (3a-3g) showed, albeit moderate, antiviral activity with $\mathrm{EC}_{50}$ values of 14 to $85 \mu \mathrm{M}$, which indicates that the novel 'enol-keto' scaffold indeed mimic the chelating function of the DKAs. Structure-activity relationship was clear in that hydroxychromone derivatives (3a-3e)
Table 1. Anti-HCV activity and cytostatic effect of 6-arylmethyloxy5-hydroxy-7-phenyl-chromone (3) derivatives ${ }^{a}$

\begin{tabular}{ccccc}
\hline Compd & $\mathrm{R}$ & $\mathrm{EC}_{50}(\mu \mathrm{M})^{b, c}$ & $\mathrm{CC}_{50}(\mu \mathrm{M})^{c, d}$ & $\mathrm{SI}^{e}$ \\
\hline 3a & $\mathrm{Cl}$ & 22 & 54 & 2.5 \\
3b & $\mathrm{Br}$ & 18 & 39 & 2.2 \\
$\mathbf{3 c}$ & $\mathrm{CN}$ & 20 & 55 & 2.8 \\
$\mathbf{3 d}$ & $\mathrm{CF}_{3}$ & 24 & 71 & 2.9 \\
$\mathbf{3 e}$ & $\mathrm{NO}_{2}$ & 14 & 35 & 2.5 \\
$\mathbf{3 f}$ & $\mathrm{Me}$ & 85 & 45 & 0.5 \\
$\mathbf{3 g}$ & $\mathrm{OMe}$ & 85 & 45 & 0.5 \\
$\mathbf{1}^{f}$ & - & $23 \pm 8$ & - & - \\
\hline
\end{tabular}

${ }^{a}$ Interferon $\alpha-2 b$ was used as a reference compound at 10000 units/well and reduced the signal in the viral RNA (luciferase) assay to background levels without any cytotoxic activity. ${ }^{b}$ Concentration required to inhibit HCV RNA replication by $50 \% .{ }^{c}$ The values obtained as the average of triplicate determinations. ${ }^{d}$ Concentration required to reduce cell proliferation by $50 \%$. ${ }^{e}$ Selectivity index: ratio of $\mathrm{CC}_{50}$ to $\mathrm{EC}_{50} .{ }^{f}$ Reference 10.

with electron withdrawing substituents $\left(\mathrm{Cl}, \mathrm{Br}, \mathrm{CN}, \mathrm{CF}_{3}, \mathrm{NO}_{2}\right)$ were more potent than those ( $\mathbf{3} \mathbf{f}$ and $\mathbf{3 g}$ ) with electron donating groups $(\mathrm{Me}, \mathrm{OMe})$. In particular, the two substituents with similar size but different electronic property, $\mathrm{CF}_{3}$ and $\mathrm{CH}_{3}$, provided the resulting hydroxychromone derivatives $\mathbf{3 d}$ and 3f, respectively, with significantly different $\mathrm{EC}_{50}$ values (24 $\mu \mathrm{M}$ and $85 \mu \mathrm{M}$, respectively). In contrast, among the series of electron withdrawing group-substituted derivatives (3a-3e), the structure-activity relationship was not clear. Also, the hydroxychromone derivatives showed similar cytostatic effect against the hepatoma cell line regardless of type of the substituent $\left(\mathrm{CC}_{50}\right.$ values, Table 1).

Taking the low solubility associated with the common chromone scaffold into account, lack of potent antiviral activity in cell-based assay as well as complicated structure-activity re- 
lationship might be attributed to the suboptimal physicochemical property of the hydroxychromone derivatives which limits its effective concentration inside the cell. In this respect, it is worth to note that DKAs also suffer from unfavorable physicochemical property, ${ }^{9}$ resulting in low antiviral activity in cell-based assay. ${ }^{10}$ However, with the proven mechanism for inhibition of the target viral enzyme, extensive structure-activity relationship around the metal-binding unit of DKA was performed to improve its unfavorable physicochemical as well as pharmacokinetic property. ${ }^{16,21}$ By the same token, optimization of the antiviral activity of the novel 'enol-keto' metal chelators is anticipated by structural modification of the hydroxychromone scaffold.

In summary, in this proof-of-concept study of finding an alternative to DKA, we observed moderate but significant inhibition of $\mathrm{HCV}$ replication $\left(\mathrm{EC}_{50}=14 \sim 85 \mu \mathrm{M}\right)$ by 5-hydroxychromone derivatives in cell-based assay. This result proposes the novel 'enol-keto' scaffold in hydroxychromone as a novel isostere for DKA and warrants extensive structure-activity relationship study for optimization of the physicochemical property and thereby antiviral activity of the 'enol-keto' metal chelators.

\section{Experimental Section}

General procedure for 6-arylmethyloxy-5-hydroxy-7-phenyl-chromen-4-one (3a-3g) synthesis. To a solution of 11 (60 $\mathrm{mg}, 0.24 \mathrm{mmol}$ ) and $\mathrm{K}_{2} \mathrm{CO}_{3}(30 \mathrm{mg}, 0.22 \mathrm{mmol})$ in DMF (5 mL) was added arylmethyl bromide $(0.22 \mathrm{mmol})$. The reaction mixture was stirred at room temperature for $4 \mathrm{~h}$. The resulting solution filtered with acetone and concentrated under reduced pressure. The crude compound was purified by column chromatography on silica gel (Hexanes:Acetone $=2: 1$ ) to afford 3a-3g.

6-(3-Chloro-benzyloxy)-5-hydroxy-7-phenyl-chromen-4one (3a): $67 \%$ yield as a white powder: ${ }^{1} \mathrm{H} \mathrm{NMR}(400 \mathrm{MHz}$, DMSO- $\left.d_{6}\right) \delta 8.07(\mathrm{~d}, J=5.8 \mathrm{~Hz}, 1 \mathrm{H}), 7.70(\mathrm{~s}, 1 \mathrm{H}), 7.53-7.40$ $(\mathrm{m}, 6 \mathrm{H}), 7.10(\mathrm{~d}, J=7.5 \mathrm{~Hz}, 2 \mathrm{H}), 5.98(\mathrm{~d}, J=5.8 \mathrm{~Hz}, 1 \mathrm{H}), 5.38$ (s, 2H); ${ }^{13} \mathrm{C}$ NMR (100 MHz, DMSO-d $\left.{ }_{6}\right) \delta 175.9,154.6,152.0$, 151.4, 142.5, 139.0, 137.5, 133.6, 130.7, 129.8, 128.4, 128.0, 127.9, 127.3, 126.7, 126.3, 116.7, 113.2, 101.2, 69.6; LC/MS (ESI) $m / z$ Found: $379.3(\mathrm{M}+\mathrm{H})^{+}$; Calcd for $\mathrm{C}_{22} \mathrm{H}_{15} \mathrm{ClO}_{4}: 378.1$.

6-(3-Bromo-benzyloxy)-5-hydroxy-7-phenyl-chromen-4one (3b): 75\% yield as a white powder: ${ }^{1} \mathrm{H}$ NMR (400 MHz, DMSO- $\left.d_{6}\right) \delta 8.72(\mathrm{~s}, 1 \mathrm{H}), 8.07(\mathrm{~d}, J=5.9 \mathrm{~Hz}, 1 \mathrm{H}), 7.83(\mathrm{~s}$, $1 \mathrm{H}), 7.57-7.54(\mathrm{~m}, 2 \mathrm{H}), 7.40-7.36(\mathrm{~m}, 1 \mathrm{H}), 7.32-7.25(\mathrm{~m}$, $4 \mathrm{H}), 7.10-7.08(\mathrm{~m}, 2 \mathrm{H}), 5.98(\mathrm{~d}, J=5.9 \mathrm{~Hz}, 1 \mathrm{H}), 5.37(\mathrm{~s}, 2 \mathrm{H})$; ${ }^{13} \mathrm{C}$ NMR $\left(100 \mathrm{MHz}, \mathrm{DMSO}-d_{6}\right) \delta 174.3,153.0,152.9,150.4$, $149.8,140.8,137.6,135.9,129.6,129.4,129.2$, 129.1, 128.2, 125.7, 125.4, 124.7, 120.5, 115.1, 111.5, 99.6; LC/MS (ESI) $m / z$ Found: $423.2(\mathrm{M}+\mathrm{H})^{+}$; Calcd for $\mathrm{C}_{22} \mathrm{H}_{15} \mathrm{BrO}_{4}$ : 422.0 .

3-(5-Hydroxy-4-oxo-7-phenyl-4H-chromen-6-yloxymethyl)-benzonitrile (3c): $71 \%$ yield as a white powder: ${ }^{1} \mathrm{H}$ NMR $\left(400 \mathrm{MHz}, \mathrm{DMSO}-d_{6}\right) \delta 8.36(\mathrm{~s}, 1 \mathrm{H}), 8.29(\mathrm{~d}, J=8.3 \mathrm{~Hz}, 1 \mathrm{H})$, 8.11-8.04 (m, 2H), $7.86(\mathrm{t}, J=7.8 \mathrm{~Hz}, 1 \mathrm{H}), 7.58-7.47(\mathrm{~m}, 4 \mathrm{H})$, 7.39-7.32 (m, 2H), $6.22(\mathrm{~d}, J=5.9 \mathrm{~Hz}, 1 \mathrm{H}), 5.63(\mathrm{~s}, 2 \mathrm{H}) ;{ }^{13} \mathrm{C}$ NMR $\left(100 \mathrm{MHz}, \mathrm{DMSO}-d_{6}\right) \delta 176.0,154.6,152.1,151.3,142.4$, 138.1, 137.5, 132.7, 132.1, 131.6, 130.0, 129.8, 127.5, 127.3, 126.4, 119.0, 116.8, 113.2, 111.8, 101.3, 69.4; LC/MS (ESI) $m / z$ Found: $370.3(\mathrm{M}+\mathrm{H})^{+}$; Calcd for $\mathrm{C}_{22} \mathrm{H}_{15} \mathrm{NO}_{4}$ : 369.1 .

5-Hydroxy-6-(3-nitro-benzyloxy)-7-phenyl-chromen-4one (3d): $79 \%$ yield as a white powder: ${ }^{1} \mathrm{H}$ NMR $(400 \mathrm{MHz}$, DMSO- $\left.d_{6}\right) \delta 8.48(\mathrm{~s}, 1 \mathrm{H}), 8.23-8.20(\mathrm{~m}, 1 \mathrm{H}), 8.08(\mathrm{~d}, J=5.9$ $\mathrm{Hz}, 1 \mathrm{H}), 8.05-8.03(\mathrm{~m}, 1 \mathrm{H}), 7.75-7.71(\mathrm{~m}, 1 \mathrm{H}), 7.32-7.25$ (m, 4H), 7.11-7.09 (m, 2H), $5.99(\mathrm{~d}, J=5.9 \mathrm{~Hz}, 1 \mathrm{H}), 5.51(\mathrm{~s}, 2 \mathrm{H})$; ${ }^{13} \mathrm{C}$ NMR $\left(100 \mathrm{MHz}\right.$, DMSO- $\left.d_{6}\right) \delta 176.0,154.7,152.0,151.3$, $148.3,142.5,138.8,137.5,134.7,130.4,129.8,127.3,126.4$, 126.3, 123.4, 122.9, 116.8, 113.2, 101.3, 69.3; LC/MS (ESI) $m / z$ Found: $390.3(\mathrm{M}+\mathrm{H})^{+}$; Calcd for $\mathrm{C}_{22} \mathrm{H}_{15} \mathrm{NO}_{6}: 389.1$.

5-Hydroxy-7-phenyl-6-(3-trifluoromethyl-benzyloxy)-chromen-4-one (3e): $71 \%$ yield as a white powder: ${ }^{1} \mathrm{H}$ NMR (400 MHz, DMSO-d $d_{6} \delta 8.75(\mathrm{~s}, 1 \mathrm{H}), 8.07(\mathrm{~d}, J=7.3 \mathrm{~Hz}, 1 \mathrm{H}), 7.99$ $(\mathrm{s}, 1 \mathrm{H}), 7.87(\mathrm{~d}, J=9.4 \mathrm{~Hz}, 1 \mathrm{H}), 7.72(\mathrm{~d}, J=9.7 \mathrm{~Hz}, 1 \mathrm{H}), 7.68-$ $7.66(\mathrm{~m}, 1 \mathrm{H}), 7.32-7.25(\mathrm{~m}, 4 \mathrm{H}), 7.11-7.09(\mathrm{~m}, 2 \mathrm{H}), 5.99(\mathrm{~d}$, $J=7.3 \mathrm{~Hz}, 1 \mathrm{H}), 5.46(\mathrm{~s}, 2 \mathrm{H}) ;{ }^{13} \mathrm{C}$ NMR $\left(100 \mathrm{MHz}, \mathrm{DMSO}-d_{6}\right)$ $\delta 175.5,154.4,151.6,151.0,142.0,137.5,137.1,132.7,131.9$, 131.8, 129.6, 129.5, 126.8, 125.9, 124.7, 124.4, 116.3, 112.8, 112.6, 100.9, 69.6; LC/MS (ESI) $m / z$ Found: $413.3(\mathrm{M}+\mathrm{H})^{+}$; Calcd for $\mathrm{C}_{23} \mathrm{H}_{15} \mathrm{~F}_{3} \mathrm{O}_{4}$ : 412.1 .

5-Hydroxy-6-(3-methyl-benzyloxy)-7-phenyl-chromen-4one (3f): $71 \%$ yield as a white powder: ${ }^{1} \mathrm{H}$ NMR $(400 \mathrm{MHz}$, DMSO- $\left.d_{6}\right) \delta$ 8.05-8.04 $(\mathrm{m}, 1 \mathrm{H}), 7.38-7.24(\mathrm{~m}, 7 \mathrm{H}), 7.17-7.15$ (m, 1H), 7.10-7.08 (m, 2H), $5.97(\mathrm{~d}, J=5.9 \mathrm{~Hz}, 1 \mathrm{H}), 5.32$ (s, 2H), 2.33 (s, 3H); ${ }^{13} \mathrm{C}$ NMR (100 MHz, DMSO- $\left.d_{6}\right) \delta 174.3$, 153.0, 150.4, 150.0, 140.9, 136.4, 135.9, 135.4, 134.7, 128.2, 127.5, 127.1, 125.7, 124.7, 124.5, 123.6, 114.9, 111.5, 99.6, 69.0, 19.7; LC/MS (ESI) $m / z$ Found: $359.3(\mathrm{M}+\mathrm{H})^{+}$; Calcd for $\mathrm{C}_{23} \mathrm{H}_{18} \mathrm{O}_{4}: 358.1$.

5-Hydroxy-6-(3-methoxy-benzyloxy)-7-phenyl-chromen4-one (3g): 63\% yield as a white powder: ${ }^{1} \mathrm{H}$ NMR $(400 \mathrm{MHz}$, DMSO- $\left.d_{6}\right) \delta 8.06-8.04(\mathrm{~m}, 1 \mathrm{H}), 7.34-7.24(\mathrm{~m}, 5 \mathrm{H}), 7.15-7.08$ (m, 4H), 6.92-6.90 (m, 1H), 6.00-5.97 (m, 1H), $5.34(\mathrm{~s}, 2 \mathrm{H})$, $3.77(\mathrm{~s}, 3 \mathrm{H}) ;{ }^{13} \mathrm{C}$ NMR $\left(100 \mathrm{MHz}, \mathrm{DMSO}-d_{6}\right) \delta 176.0,159.8$, 154.6, 152.0, 151.5, 142.5, 138.0, 137.5, 130.0, 129.8, 127.3, 126.4, 126.2, 120.1, 116.6, 113.9, 113.7, 113.1, 101.3, 70.4, 55.4; LC/MS (ESI) $m / z$ Found: $375.3(\mathrm{M}+\mathrm{H})^{+}$; Calcd for $\mathrm{C}_{23} \mathrm{H}_{18} \mathrm{O}_{5}$ : 374.1 .

Anti-HCV assay. The human hepatoma cell line Huh-5-2 carrying the subgenomic HCV genotype 1 replicon with the luc-ubi-neo (reporter/selective) fusion gene, was kindly provided by Dr. Ralf Bartenschlager (University of Heidelberg, Heidelberg, Germany). Huh-5-2 cells were seeded at a density of $5 \times 10^{3}$ per well in a tissue culture-treated white 96-well view plate in complete DMEM supplemented with $500 \mu \mathrm{g} / \mathrm{mL} \mathrm{G418.}$ After incubation for $24 \mathrm{~h}$ at $37{ }^{\circ} \mathrm{C}\left(5 \% \mathrm{CO}_{2}\right)$, medium was refreshed (with G418) and DMSO stock of test compounds were added. After 4 days of incubation at $37^{\circ} \mathrm{C}$, cell culture medium was removed and luciferase activity was determined using the Steady-Glo luciferase assay system (Promega, Leiden, The Netherlands).

Cytostatic effect. Huh-5-2 cells were seeded at a density of $5 \times 10^{3}$ per well of a 96-well plate in complete DMEM with the appropriate concentrations of G418. Serial dilutions of the test compounds in complete DMEM without G418 were added $24 \mathrm{~h}$ after seeding. Cells were allowed to proliferate for 3 days at $37{ }^{\circ} \mathrm{C}$, after which the cell number was determined by WST-1 assay. 
Acknowledgments. This work was supported by a grant of the Korea Healthcare technology R\&D Project, Ministry for Health, Welfare \& Family Affairs, Republic of Korea (A084628-AA2023-08N1-00010A), a grant from the Korean Rural Development Administration [Agenda program, (RDA, 11-3068)], Biogreen 21 (Korea Ministry of Agriculture and Forestry), and Priority Research Centers Program through the National Research Foundation of Korea (NRF) funded by the Ministry of Education, Science and Technology (2009-0093824).

\section{References}

1. Choo, Q. L.; Kuo, G.; Weiner, A. J.; Overby, L. R.; Bradley, D. W.; Houghton, M. Science 1989, 244, 359.

2. Subramanian, G. M.; Fiscella, M.; Lamouse-Smith, A.; Zeuzem, S.; McHutchison, J. G. Nat. Biotech. 2007, 25, 1411.

3. Manns, M. P.; Foster, G. R.; Rockstroh, J. K.; Zeuzem, S.; Zoulim, F.; Houghton, M. Nat. Rev. Drug Discov. 2007, 6, 991.

4. De Francesco, R.; Tomei, L.; Altamura, S.; Summa, V.; Migliaccio, G. Antiviral Res. 2003, 58, 1 .

5. Summa, V.; Petrocchi, A.; Pace, P.; Matassa, V. G.; De Francesco, R.; Altamura, S.; Tomei, L.; Koch, U.; Neuner, P. J. Med. Chem. 2004, 47, 14

6. Kim, J.; Han, J. H.; Chong, Y. Bull. Korean Chem. Soc. 2006, 27 , 1919.

7. Kim, J.; Chong, Y. Mol. Simul. 2006, 32, 1131.

8. Kim, J.; Kim, K.-S.; Lee, H. S.; Park, K.-S.; Park, S. Y.; Kang, S.-Y.; Lee, S. J.; Park, H. S.; Kim, D.-E.; Chong, Y. Bioorg. Med. Chem. Lett. 2008, 18, 4661.

9. Summa, V.; Petrocchi, A.; Matassa, V. G.; Gardelli, C.; Muraglia, E.; Rowley, M.; Paz, O. G.; Laufer, R.; Monteagudo, E.; Pace, P. J. Med. Chem. 2006, 49, 6646.

10. Paeshuyse, J.; Vliegen, I.; Coelmont, L.; Leyssen, P.; Tabarrini, O.; Herdewijn, P.; Mittendorfer, H.; Easmon, J.; Cecchetti, V.; Bartenschlager, R.; Puerstinger, G.; Neyts, J. Antimicrob. Agents Chemother. 2008, 52, 3433.

11. Barreca, M. L.; Ferro, S.; Rao, A.; Luca, L. D.; Zappala, M.; Monforte, A. M.; Debyser, Z.; Witvrouw, M.; Chimirri, A. J. Med. Chem. 2005, 48, 7084 .
12. Goldgur, Y.; Craigie, R.; Cohen, G. H.; Fujiwara, T.; Yoshinage, T.; Fujishita, T.; Sugimoto, H.; Endo, T.; Murai, H.; Davies, D. R. Proc. Natl. Acad. Sci. USA 1999, 96, 13040.

13. Zhuang, L.; Wai, J. S.; Embrey, M. W.; Fisher, T. E.; Egbertson, M. S.; Payne, L. S.; Guare, J. P., Jr.; Vacca, J. P.; Hazuda, D. J.; Felock, P. J.; Wolfe, A. L.; Stillmock, K. A.; Witmer, M. V.; Moyer, G.; Schleif, W. A.; Gabryelski, L. J.; Leonard, Y. M.; Lynch, J. J., Jr.; Michelson, S. R.; Young, S. D. J. Med. Chem. 2003, 46, 453.

14. Hazuda, D. J.; Anthony, N. J.; Gomez, R. P.; Jolly, S. M.; Wai, J. S.; Zhuang, L.; Fisher, T. E.; Embrey, M.; Guare, J. P., Jr.; Egbertson, M. S.; Vacca, J. P.; Huff, J. R.; Felock, P. J.; Witmer, M. V.; Stillmock, K. A.; Danovich, R.; Grobler, J.; Miller, M. D.; Espeseth, A. S.; Jin, L.; Chen, I.-W.; Lin, J. H.; Kassahun, K.; Ellis, J. D.; Wong, B. K.; Xu, W.; Pearson, P. G.; Schleif, W. A.; Cortese, R.; Emini, E.; Summa, V.; Holloway, M. K.; Young, S. D. Proc. Natl. Acad. Sci. USA 2004, 101, 11233.

15. Hazuda, D. J.; Young, S. D.; Guare, J. P.; Anthony, N. J.; Gomez, R. P.; Wai, J. S.; Vacca, J. P.; Handt, L.; Motzel, S. L.; Klein, H. J.; Dornadula, G.; Danovich, R. M.; Witmer, M. V.; Wilson, K. A. A.; Tussey, L.; Schleif, W. A.; Gabryelski, L. S.; Jin, L.; Miller, M. D.; CAsimiro, D. R.; Emini, E. A.; Shiver, J. W. Science 2004, 305, 528.

16. Summa, V.; Petrocchi, A.; Bonelli, F.; Crescenzi, B.; Donghi, M.; Ferrara, M.; Fiore, F.; Gardelli, C.; Paz, O. G.; Hazuda, D. J.; Jones, P.; Kinzel, O.; Laufer, R.; Monteagudo, E.; Muraglia, E.; Nizi, E.; Orvieto, F.; Pace, P.; Pescatore, G.; Scarpelli, R.; Stillmock, K.; Witmer, M. V.; Rowley, M. J. Med. Chem. 2008, 51, 5843.

17. Sato, M.; Kawakami, H.; Motomura, T.; Aramaki, H.; Matsuda, T.; Yamashita, M.; Ito, Y.; Matsuzaki, Y.; Yamataka, K.; Ikeda, S.; Shinkai, H. J. Med. Chem. 2009, 52, 4869.

18. Lohmann, V.; Korner, F.; Koch, J.; Herian, U.; Theilmann, L.; Bartenschlager, R. Science 1999, 285, 110.

19. Vroljk, J. M.; Kaul, A.; Hansen, B. E.; Lohmann, V.; Haagmans, B. L.; Schalm, S. W.; Bartenschlager, R. J. Virol. Methods 2003, 110,201

20. Gozdek, A.; Zhukov, I.; Polkowska, A.; Poznanski, J.; Stankiewicz-Drogon, A.; Pawlowicz, J. M.; Zagorski-Ostoja, W.; Borowski, P.; Boguszewska-Chachulska, A. Antimicrob. Agents Chemother. 2008, 52, 393.

21. Sato, M.; Kawakami, H.; Motomura, T.; Aramaki, H.; Matsuda, T.; Yamashita, M.; Ito, Y.; Matsuzaki, Y.; Yamataka, K.; Ikeda, S.; Shinkai, H. J. Med. Chem. 2009, 52, 4869. 